\title{
Measuring the burden of disease using disability-adjusted life years in Shilin County of Yunnan Province, China
}

\author{
Shang-Cheng Zhou $\cdot$ Le Cai $\cdot$ Jing Wang $\cdot$ \\ Shao-guo Cui · Yun Chai · Bing Liu • \\ Chong-Hua Wan
}

Received: 22 April 2010/Accepted: 4 August 2010/Published online: 4 September 2010

(C) The Japanese Society for Hygiene 2010

\begin{abstract}
Background Few studies calculating burden of disease (BOD) have been carried out in China. Disability-adjusted life years (DALY) is one of the useful methods used to estimate BOD. This study aims to use DALY for evaluating BOD and to provide useful information for health planning for residents in Shilin Yi Nationality Autonomous County (Shilin County) of Yunnan Province, China.

Methods Methods developed for the Global Burden of Disease (GBD) Study by the World Bank and World Health
\end{abstract}

S.-C. Zhou · J. Wang · S. Cui · Y. Chai · B. Liu

Faculty of Public Administration,

Yunyang Medical College, Shiyan 442000,

People's Republic of China

e-mail: zsc_2k@163.com

J. Wang

e-mail: 547376316@qq.com

S. Cui

e-mail: 112420225@qq.com

Y. Chai

e-mail: 1286886429@qq.com

B. Liu

e-mail: xsky2008@163.com

\section{S.-C. Zhou}

School of Medicine and Health Management,

Tongji Medical College,

Huazhong University of Science and Technology,

Wuhan 430030, People's Republic of China

S.-C. Zhou · L. Cai · C.-H. Wan ( $₫)$

Faculty of Public Health, Kunming Medical College,

Kunming 650031, People's Republic of China

e-mail:whzsc2008@hotmail.com

L. Cai

e-mail: zsc2000@21cn.com
Organization (WHO) were adapted and applied to Shilin County population health data. DALY rate per 1,000 was calculated from medical death certificates in 2003 in Shilin County. The geographic coordinates of towns or townships were determined using the geocode function of R2.3.1 geographical information system (GIS) software.

Results Respiratory diseases were by far the leading cause of years of life lost (YLL) in both males and females. The four other leading causes of YLL in descending order were: unintentional injuries, cardiovascular diseases, intentional injuries, and malignant neoplasms. However, the five leading causes of years lived with disability (YLD) were, in descending order: neuropsychiatric conditions, intentional injuries, respiratory diseases, unintentional injuries, and cardiovascular diseases. The leading cause of total disease burden (DALY) was neuropsychiatric conditions. Townships of Muzhuqing, Xijiekou, and Weize were the areas with most serious disease burden in Shilin County.

Conclusions Prevention and treatment of neuropsychiatric conditions and respiratory diseases for both females and males should be enhanced in Shilin County, so as to decrease injury. More preventive interventions for noninfectious chronic diseases should be emphasized in remote townships.

Keywords Burden of disease - Disability adjusted life years · All-causes-of-death survey data · Geographical information system

\section{Background}

In the current situation with increasing threat of chronic diseases and emerging and re-emerging infectious diseases, how to allocate limited health resources is always a common serious problem for the World Health Organization 
(WHO) and each nation [1, 2]. The study of burden of disease (BOD) provides important evidence for setting priorities and emphases in health research, and favors optimization of allocation of health resources and improving human quality of life. Valid health statistics are required for policy-making and taking essential measures. With the development of society, the sequence of disease and causes of death are changing. People have realized that health outcomes are not only fatal but also include nonfatal situations and disability in different degrees. Traditional indexes such as mortality rate, prevalence or incidence are limited and unilateral and cannot value severe diseases' effect on health. So, more sensitive measures are needed to study overall health and BOD [3]. Study of BOD was based on use of a single death indicator for evaluating BOD until the adoption of certain compound indicators considering disability and death. During this process, many indicators have emerged sequentially, including morbidity rate, mortality rate, years of potential life lost (YPLL), disability-adjusted life years (DALY), and so on $[4,5]$.

Shilin Yi Nationality Autonomous County (Shilin County) is a rural region in the southeast of Kunming City, Yunnan Province [6, 7]. It is $78 \mathrm{~km}$ from the main urban area of Kunming and covers a land area of $1,719 \mathrm{~km}^{2}$. Its permanent population amounts to 230,548 (116,204 males, 114,344 females) in 2003, wherein Yi population account for $34 \%$ [8, 9]. Average life expectancy in Shilin County was 70.12 years for males and 73.89 years for females [10].

The Shilin County burden of disease and injury studies were the first comprehensive studies of this type in Yunnan Province. The study used methods developed for the Global Burden of Disease Study (GBD) [11] to quantify loss of health from a comprehensive set of 135 causes of disease and injury. It used a common metric of DALY, which combines information on both the impact of premature death and the impact of disability and other nonfatal health outcomes. One DALY can be thought of as one lost year of "healthy" life, and the burden of disease can be considered as a measurement of the gap between current health status and an ideal situation of living into old age free of disease and disability. DALY has previously been used to provide a comprehensive assessment of global burden of disease and injury for the World Bank [12], to inform global priority setting for health research [13], and to report on trends in population health across the world [14].

\section{Methods}

Study population

Shilin County was selected as the study region. There were 1,065 deaths (563 males and 502 females) in the region.
Data source

Causes of death were based on medical death certificate information, maintained by Shi lin Center for Disease Control and Prevention (CDC). All death reports were grouped by underlying cause of death as defined in the GBD study [15] and coded using the International Classification of Diseases, 9th revision (ICD-9) coding system.

To avoid misreporting of age at death or misclassification of cause of death, all medical death certificates were verified for underlying cause of death by a team of two independent physicians. Any discrepancies were reviewed to obtain consensus. All death conditions were distributed to other more well-defined causes according to the age and gender distribution of specific conditions, following the conceptual approach in the GBD study [16]. These were subdivided into several more specific causes [16]. Because ill-defined causes were exist, 50 deaths of ill-defined causes were beyond this analysis.

\section{Calculation of YLL}

Premature mortality was estimated in terms of YLL using the widely used formula [17-19]. To calculate YLL, the GBD DALY template was used in this study [20]. This is a Microsoft Excel spreadsheet that contains the formula to calculate YLL.

\section{Calculation of years lived with disability and DALY}

The GBD formula was applied for calculation of DALY. DALY was calculated by summing YLL and YLD:

$\mathrm{DALY}=\mathrm{YLL}+\mathrm{YLD}$.

DALY was calculated using both a $3 \%$ discount rate and no discounting, although in this paper we only report discounted estimates. Death estimates alone were the data source for YLL computation. Prevalence estimates, together with disability weighting data, were used to calculate YLD. Because we are short of incidence, prevalence, even sequelae of disease and injury, we use the indirect method. We can estimate YLD and DALY indirectly according to death register data, demographic data, and the YLD/YLL ratio for China taken from the WHO World Health Report 2002.

Geographical information system (GIS) methodology

Geographic information system (GIS) mapping technologies have potential to advance public health promotion by mapping regional differences in attributes to suggest priorities for public health interventions. The geographic 
Table 1 Distribution of deaths using mortality and DALY by age and sex in Shilin County (2003)

\begin{tabular}{|c|c|c|c|c|c|c|}
\hline \multirow[t]{2}{*}{ Age group } & \multicolumn{2}{|l|}{ Male } & \multicolumn{2}{|l|}{ Female } & \multicolumn{2}{|l|}{ Total } \\
\hline & DALY/1,000 & Mortality (\%o) & DALY/1,000 & Mortality (\%o) & DALY/1,000 & Mortality (\%) \\
\hline $0 \sim$ & 1,309.0 & 39.53 & $1,009.0$ & 30.37 & $1,164.1$ & 35.11 \\
\hline $1 \sim$ & 164.8 & 4.69 & 117.8 & 3.34 & 142.0 & 4.03 \\
\hline $5 \sim$ & 32.9 & 0.88 & 17.2 & 0.46 & 25.2 & 0.68 \\
\hline $10 \sim$ & 22.3 & 0.60 & 31.6 & 0.84 & 26.8 & 0.72 \\
\hline $15 \sim$ & 24.2 & 0.67 & 39.8 & 1.10 & 31.4 & 0.87 \\
\hline $20 \sim$ & 54.8 & 1.62 & 51.8 & 1.52 & 53.4 & 1.57 \\
\hline $25 \sim$ & 43.8 & 1.41 & 33.2 & 1.06 & 38.8 & 1.24 \\
\hline $30 \sim$ & 71.0 & 2.53 & 25.6 & 0.90 & 49.1 & 1.75 \\
\hline $35 \sim$ & 56.9 & 2.29 & 44.8 & 1.77 & 51.2 & 2.04 \\
\hline $40 \sim$ & 82.8 & 3.82 & 44.9 & 2.03 & 64.5 & 2.96 \\
\hline $45 \sim$ & 64.8 & 3.50 & 53.1 & 2.79 & 59.0 & 3.15 \\
\hline $50 \sim$ & 100.2 & 6.44 & 67.7 & 4.17 & 84.4 & 5.34 \\
\hline $55 \sim$ & 144.3 & 11.32 & 50.2 & 3.75 & 99.2 & 7.69 \\
\hline $60 \sim$ & 209.1 & 20.69 & 95.7 & 8.77 & 154.2 & 14.93 \\
\hline $65 \sim$ & 221.4 & 28.69 & 160.2 & 18.77 & 191.1 & 23.78 \\
\hline $70 \sim$ & 263.2 & 46.28 & 220.9 & 34.52 & 241.9 & 40.35 \\
\hline $75 \sim$ & 296.5 & 73.87 & 246.0 & 53.89 & 269.0 & 63.00 \\
\hline $80 \sim$ & 396.8 & 147.40 & 306.1 & 99.64 & 344.0 & 119.58 \\
\hline $85 \sim$ & 293.5 & 201.52 & 264.8 & 172.79 & 275.2 & 183.20 \\
\hline
\end{tabular}

coordinates of towns or townships were determined using the geocode function of R2.3.1 geographical information system (GIS) software. Addresses that did not automatically match during batch processing with R2.3.1 were geocoded manually using detailed paper street maps [21].

Ethical approval

This study was approved by the Ethics Committee of Kunming Medical College, before carrying out the research.

\section{Results}

Table 1 presents the distribution of deaths using mortality and DALY by age and sex in Shilin County (2003). Measuring on different indexes of the mortality or DALY, the leading age groups of disease burden were different. The mortality of age groups increased gradually with age increasing by no weight, whereas DALY emphasized the weight of young people and weakened the weight of elder.

Respiratory diseases were by far the leading cause of years of life lost in both males and females, followed by unintentional injuries, cardiovascular diseases, intentional injuries, and malignant neoplasms (Table 2).

Neuropsychiatric conditions were the leading cause of YLD. These were followed by intentional injuries and
Table 2 YLL by sex and broad disease groups in Shilin County (2003)

\begin{tabular}{lrlr}
\hline Male & YLL & Female & YLL \\
\hline Respiratory diseases & 1,605 & Respiratory diseases & 1,567 \\
Unintentional injuries & 1,545 & Cardiovascular diseases & 1,193 \\
Cardiovascular diseases & 995 & Intentional injuries & 840 \\
Intentional injuries & 905 & Unintentional injuries & 679 \\
Malignant neoplasms & 863 & Malignant neoplasms & 571 \\
Maternal conditions & 697 & Maternal conditions & 397 \\
Digestive diseases & 304 & Respiratory infections & 320 \\
Infectious and parasitic & 276 & Infectious and parasitic & 181 \\
$\quad$ diseases & & diseases & \\
Respiratory infections & 275 & Congenital anomalies & 120 \\
Neuropsychiatric & 217 & Digestive diseases & 90 \\
$\quad$ conditions & & & \\
Congenital anomalies & 171 & Genito-urinary diseases & 85 \\
Genito-urinary diseases & 129 & Neuropsychiatric & 70 \\
& & conditions & \\
Diabetes mellitus & 79 & Diabetes mellitus & 20 \\
Uncertain cause & 122 & Uncertain cause & 141 \\
Total & 8,183 & Total & 6,274 \\
\hline
\end{tabular}

respiratory diseases. In terms of specific conditions, neuropsychiatric conditions were the leading cause of nonfatal disease burden in Shilin County, causing 48.5 YLD/1,000 in 2003 (Table 3). 
Table 3 YLL/1,000, YLD/1,000, and DALY/1,000 population by broad disease groups in Shilin County (2003)

\begin{tabular}{lrrr}
\hline All causes & YLL & YLD & DALY \\
\hline Infectious and parasitic diseases & 2.1 & 1.4 & 3.5 \\
Respiratory infections & 2.7 & 0.5 & 3.2 \\
Respiratory diseases & 14.2 & 6.6 & 20.8 \\
Maternal conditions & 4.9 & 1.3 & 6.2 \\
Malignant neoplasms & 6.4 & 1.7 & 8.1 \\
Diabetes mellitus & 0.4 & 0.5 & 0.9 \\
Neuropsychiatric conditions & 1.3 & 48.5 & 49.8 \\
Cardiovascular diseases & 9.8 & 2.2 & 12.0 \\
Digestive diseases & 1.8 & 1.1 & 2.9 \\
Genito-urinary diseases & 1.0 & 0.7 & 1.7 \\
Congenital anomalies & 1.3 & 0.7 & 2.0 \\
Unintentional injuries & 9.9 & 4.8 & 14.7 \\
Intentional injuries & 7.8 & 13.2 & 21.0 \\
Total & 63.6 & 59.1 & 122.7 \\
\hline
\end{tabular}

Table 4 Ordering of towns and townships in Shilin County by disease burden (DALY/1,000) (2003)

\begin{tabular}{llll}
\hline Serial number & District & Population & DALY/1,000 \\
\hline 1 & Muzhuqing township & 12,835 & 70.9 \\
2 & Xijiekou township & 15,105 & 70.8 \\
3 & Weize township & 13,541 & 68.3 \\
4 & Lumeiyi township & 31,364 & 65.4 \\
5 & Shilin town & 12,284 & 64.7 \\
6 & Dake township & 16,947 & 59.7 \\
7 & Beidacun township & 25,104 & 53.3 \\
8 & Guishan township & 18,025 & 51.7 \\
9 & Banqiao township & 35,253 & 48.0 \\
10 & Lufu town & 50,180 & 31.0 \\
\hline
\end{tabular}

As shown in Table 4, inclusion of nonfatal health outcomes led to a substantially different picture to that provided by traditional mortality statistics: respiratory diseases became the third leading cause (20.8 DALY/1,000), after neuropsychiatric conditions $(49.8 \mathrm{DALY} / 1,000)$ and intentional injuries (21.0 DALY/1,000).

Shilin County includes 2 urban townships (Lufu town, Shilin town) and 8 rural townships (Muzhuqing township, Xijiekou township, Weize township, Lumeiyi township, Lumeiyi township, Dake township, Beidacun township, Guishan township, Banqiao township). Methodological quality tended to produce variation in different directions for different causes of deaths. Table 4 and Fig. 1 show the regional variation of disease burden by DALY in Shilin County. Townships of Muzhuqing, Xijiekou, and Weize were areas of serious disease burden.

Table 5 presents YLL/1,000, YLD/1,000, and DALY/ 1,000 population by major disease groups of the heaviest

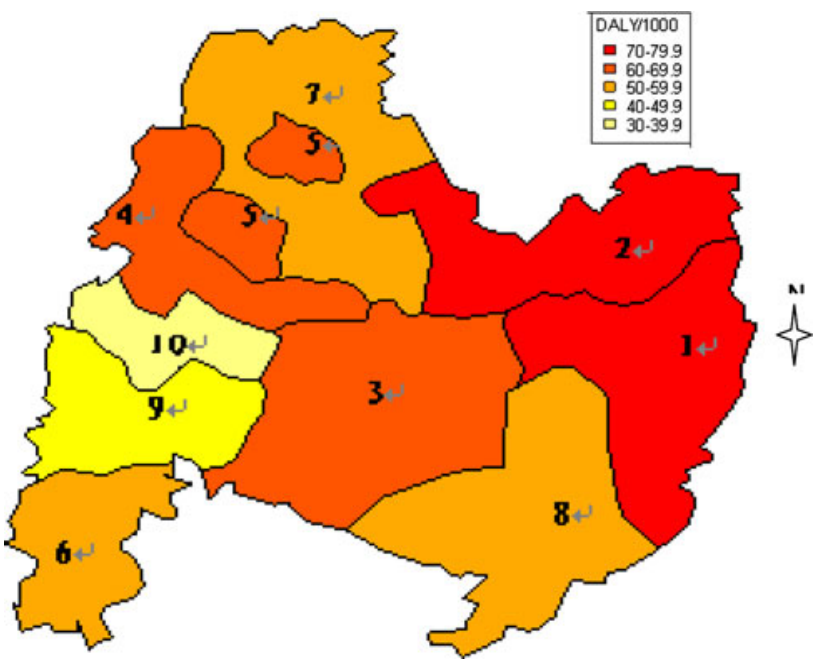

Fig. 1 Distribution of disease burden by DALY in Shilin County (2003) by towns and townships. The serial numbers $(1,2,3$, etc.) have the same meaning as in Table 3

Table 5 YLL/1,000, YLD/1,000, and DALY/1,000 population by major disease groups of the heaviest disease burden township in Shilin County (2003)

\begin{tabular}{lcll}
\hline Causes & YLL/1,000 $^{\mathrm{a}}$ & $\mathrm{YLD}^{2} 1,000^{\mathrm{a}}$ & $\mathrm{DALY} / 1,000^{\mathrm{a}}$ \\
\hline Maternal conditions & \multicolumn{1}{c}{13} & 1.3 & 14.3 \\
Unintentional injuries & 11.6 & 0.7 & 12.3 \\
Intentional injuries & 8.1 & 1.3 & 9.4 \\
Cardiovascular diseases & 8.9 & 0.4 & 9.3 \\
Respiratory diseases & 7.5 & 0.7 & 8.2 \\
\hline
\end{tabular}

${ }^{a}$ These indicators are calculated by summing Muzhuqing township and Xijiekou township

township in Shilin County (2003). The indicators above were calculated by summing Muzhuqing township and Xijiekou township because their disease burden was the heaviest. The five leading causes of DALY/1,000 in descending order were: maternal conditions, unintentional injuries, intentional injuries, cardiovascular diseases, and respiratory diseases.

\section{Discussion}

As expected, our investigation of all disease burden showed variation both by cause and within geographical regions.

Burden-of-disease analysis provides a unique perspective on health that integrates fatal and nonfatal outcomes yet allows these two classes of outcomes to be examined separately. Among the top 10 causes of burden of disease and injury in Shilin County were two nonfatal or lowfatality diseases: neuropsychiatric conditions and congenital anomalies. The burden of neuropsychiatric conditions 
and congenital anomalies had been seriously underestimated by traditional approaches to disease burden assessment that disregard disability and take into account only deaths. Especially, neuropsychiatric conditions had the highest DALY rate (49.8 DALY/1,000), similar to in another study (Shanghai, 30.52 DALY/1,000) [22].

The calculation of YLL was straightforward, and the precision of the estimates was almost entirely dependent on the quality of the data for underlying cause of death. The YLD required much more grounding data and extensive epidemiological modeling. Thus, the precision of the YLD estimates was not calculated in the usual statistical sense. In our study, we used the indirect method to calculate YLD. We estimated YLD according to death register data, demographic data, and the YLD/YLL ratio for China from the WHO World Health Report 2002. As a next step in these studies, we plan to detect the uncertainty in these burden-ofdisease estimates using simulation modeling and sensitivity analysis to calculate YLD directly and accurately.

In Shilin County, burden of disease in 2003 was about 14,457 DALY. Depending on the mortality and DALY used for calculation, rank results could be slightly different. DALY led to overestimation for young people compared with the mortality norm, decreasing the burden of disease of the elder gap. That is to say, the social and economical values were considered by DALY. Actually the GBD study weighted a year of healthy life lived at youngest ages and oldest ages lower than years lived at other ages.

Few previous studies have reported cause-specific mortality or DALY in China [23], and Shilin County is distinctive. Respiratory diseases, unintentional injuries, cardiovascular diseases, intentional injuries, and malignant neoplasms were important causes of YLL and DALY in the study region. Despite the fact that economic growth has resulted in more high-speed vehicles in China, the reduction in disease burden due to respiratory diseases indicates a positive trend in this context. As in other locations [22], high injury rates in Shilin (Shilin: unintentional injuries 14.7 DALY/1,000, intentional injuries 21.0 DALY/1,000; Shanghai: unintentional injuries 9.38 DALY/1,000) may be due to multiple factors such as religious beliefs, social isolation, family problems, stressful life events, chronic painful diseases, mental disorders, and substance abuse [24]. In this study, digestive diseases led to more premature deaths in males than females, whereas deaths due to neuropsychiatric conditions were more common in females. As such, these diseases need better control according to sex.

GIS is a geomatic tool used for geographical analysis and is crucial to understand injury distribution better. This computer mapping technology has introduced a new era in computational epidemiology. Through GIS, epidemiologists can translate or geocode street addresses into unique latitude and longitude locations [25]. In our study, the cause of heavy disease burden in the townships of Muzhuqing and Xijiekou may be mainly mountainous with high variability in level of socioeconomic development of different areas. Strategies of economic development should be reviewed to avoid adverse effects on health, especially due to maternal conditions.

A few limitations should be recognized in interpreting this study's results. The strength of this study comes from its use of complete data from death registration systems. The problem of underreporting of deaths found in many studies was thus minimized. Underreporting of deaths has been shown to be more common in infant deaths in a previous study, especially in rural regions [26]. Such work will be the focus of future research. More detailed work could be done to estimate disease burden at specific disease level, which would take account of differences in mortality outcomes.

This study is a first step in exploring the usefulness of BOD methods for Yunnan Province. Although the application of the cause-of-death evaluation framework at the county level in Shilin County produced important findings, some of these should be viewed with caution; for example, there was uncertainty regarding the validity of registration diagnoses for specified causes of death without the issue of ill-defined causes.

\section{Conclusions}

Overall, our findings for Shilin County's disease burden suggest that DALY could provide a more complex measure of the impact than traditional death rates. Prevention and treatment of neuropsychiatric conditions and respiratory diseases for both females and males should be enhanced in Shilin County, so as to decrease injuries. Prevention and intervention for noninfectious chronic diseases should be emphasized in remote townships. Additional studies are needed to determine whether these findings are true for other areas, including rural areas and more minor nationality autonomous areas.

Acknowledgments This research was supported by Shilin Health Bureau and Shilin Maternal and Children Hospital; we thank all of these sectors for providing the mortality data for this study. The study was supported by both Yunnan Provincial Natural Science Funds (grant number: 2003C0022Q) and Research Program of Humanities and Social Sciences by MOE, P.R.C. (grant number: 09YJC840042). The funding sources influenced neither the technical part of the research nor the submission of the manuscript.

Conflict of interest The authors declare that they have no competing interests.

\section{Appendix}

In this article, the YLD/YLL ratio was taken as that of China from the WHO World Health Report 2002 (Table 6). 
Table 6 YLD/YLL ratio by broad disease groups in Shilin County (2003)
Table 7 Impact of ageweighting and discounting on YLL in Shilin County (2003) $[r, K, \beta]$

\begin{tabular}{|c|c|c|c|c|}
\hline \multirow{2}{*}{$\begin{array}{l}\text { Cause } \\
\text { (1) }\end{array}$} & \multicolumn{2}{|c|}{ Shilin County } & \multicolumn{2}{|c|}{ CHN (WHO report) } \\
\hline & $\begin{array}{l}\text { YLL } \\
\text { (2) }\end{array}$ & $\begin{array}{l}\text { YLD } \\
(3)=(2) \times(5) /(4)\end{array}$ & $\begin{array}{l}\text { YLL } \\
\text { (4) }\end{array}$ & $\begin{array}{l}\text { YLD } \\
(5)\end{array}$ \\
\hline Infectious and parasitic diseases & 2.1 & 1.4 & 7.5 & 5.0 \\
\hline Respiratory infections & 2.7 & 0.5 & 4.3 & 0.76 \\
\hline Respiratory diseases & 14.2 & 6.6 & 8.4 & 3.9 \\
\hline Maternal conditions & 4.9 & 1.3 & 7.6 & 2.0 \\
\hline Malignant neoplasms & 6.4 & 1.7 & 14.3 & 3.7 \\
\hline Diabetes mellitus & 0.4 & 0.5 & 0.8 & 1.0 \\
\hline Neuropsychiatric conditions & 1.3 & 48.5 & 0.7 & 26.1 \\
\hline Cardiovascular diseases & 9.8 & 2.2 & 15.3 & 3.4 \\
\hline Digestive diseases & 1.8 & 1.1 & 4 & 2.5 \\
\hline Genito-urinary diseases & 1.0 & 0.7 & 1.3 & 0.9 \\
\hline Congenital anomalies & 1.3 & 0.7 & 2.4 & 1.3 \\
\hline Unintentional injuries & 9.9 & 4.8 & 12.5 & 6.1 \\
\hline Intentional injuries & 7.8 & 13.2 & 4.9 & 8.3 \\
\hline
\end{tabular}

\begin{tabular}{|c|c|c|c|c|c|c|}
\hline \multirow[t]{2}{*}{ Age group } & \multicolumn{3}{|l|}{ Male } & \multicolumn{3}{|l|}{ Female } \\
\hline & $0.03,1,0.04$ & $0,1,0.04$ & $0.03,0,0$ & $0.03,1,0.04$ & $0,1,0.04$ & $0.03,0,0$ \\
\hline $0 \sim$ & $1,309.0$ & $3,396.1$ & $1,198.0$ & $1,009.0$ & $2,648.4$ & 926.9 \\
\hline $1 \sim$ & 164.8 & 400.9 & 141.0 & 117.8 & 289.8 & 101.2 \\
\hline $5 \sim$ & 32.9 & 72.9 & 26.2 & 17.2 & 38.5 & 13.7 \\
\hline $10 \sim$ & 22.3 & 45.6 & 17.3 & 31.6 & 65.9 & 24.8 \\
\hline $15 \sim$ & 24.2 & 46.7 & 19.0 & 39.8 & 77.9 & 31.5 \\
\hline $20 \sim$ & 54.8 & 101.3 & 44.5 & 51.8 & 97.1 & 42.5 \\
\hline $25 \sim$ & 43.8 & 77.4 & 37.3 & 33.2 & 59.6 & 28.6 \\
\hline $30 \sim$ & 71.0 & 120.0 & 64.4 & 25.6 & 44.1 & 23.6 \\
\hline $35 \sim$ & 56.9 & 92.3 & 55.3 & 44.8 & 74.1 & 44.2 \\
\hline $40 \sim$ & 82.8 & 128.4 & 86.7 & 44.9 & 71.3 & 48.0 \\
\hline $45 \sim$ & 64.8 & 96.1 & 73.5 & 53.1 & 80.8 & 61.6 \\
\hline $50 \sim$ & 100.2 & 142.0 & 123.4 & 67.7 & 98.7 & 85.2 \\
\hline $55 \sim$ & 144.3 & 195.2 & 193.3 & 50.2 & 70.0 & 69.1 \\
\hline $60 \sim$ & 209.1 & 269.6 & 305.6 & 95.7 & 127.4 & 143.7 \\
\hline $65 \sim$ & 221.4 & 272.2 & 353.8 & 160.2 & 203.4 & 263.2 \\
\hline $70 \sim$ & 263.2 & 309.7 & 460.9 & 220.9 & 267.4 & 397.8 \\
\hline $75 \sim$ & 296.5 & 335.7 & 573.2 & 246.0 & 284.8 & 487.1 \\
\hline $80 \sim$ & 396.8 & 434.7 & 855.1 & 306.1 & 340.7 & 671.8 \\
\hline $85 \sim$ & 293.5 & 310.6 & 738.6 & 264.8 & 281.7 & 689.5 \\
\hline Total & 103.3 & 190.4 & 116.4 & 75.7 & 142.4 & 88.6 \\
\hline
\end{tabular}

Table 7 presents the impact of the use of discounting and age-weighting on the distribution of deaths by age and sex of YLL. We can see that use of discounting and age- weighting slightly modified the ranking of diseases for both males and females. That is to say, the result of this study was generally stable. 


\section{References}

1. Murray CJ, Lopez AD. Evidence-based health policy-lessons from the global Burden of Disease Study. Science. 1996; 274(5288):740-3.

2. Hense HW, Antes G. Approaches to evidence-based medicine and the cochrane collaboration. Z Kardiol. 1997;86(5):313-9.

3. Anand S, Hanson K. Disability adjusted life years: a critical review. J Health Econ. 1997;16(6):685-702.

4. Wang SY. Advance in the methods of analysis and evaluation of cause of death. Zhonghua Liu Xing Bing Xue Za Zhi. 1996; 17(4):239-42.

5. MacNab YC. Mapping disability adjusted life years: a Bayesian hierarchical model framework for burden of disease and injury assessment. Stat Med. 2007;26(26):4746-69.

6. Cai L, Virasakdi C, Alan G. Contextual socioeconomic determinants of cardiovascular risk factors in rural south-west China: a multilevel analysis. BMC Public Health. 2007;7:72.

7. Cai L, Chongsuvivatwong V, Geater A. Changing pattern of premature mortality burden over 6 years of rapid growth of the economy in suburban south-west China: 1998-2003. Public Health. 2008;122:478-86.

8. Yunnan Province Statistical Bureau. Yunnan statistical yearbook 2004. Beijing: China Statistics; 2005.

9. Zhou SC, Cai L, Wan CH, Lv YL, Fang PQ. Assessing the disease burden of Yi people by years of life lost in Shilin county of Yunnan province, China. BMC Public Health. 2009;9:188.

10. Jiahong LUO, Weihong BI, Meixian LUO. Study on life expectancy for the male and female of Shilin County in Yunnan province. Chin Rural Health Serv Admin. 2004;24(07):20-2.

11. Murray CJ, Lopez AD. The global burden of disease: a comprehensive assessment of mortality and disability from diseases, injuries and risk factors in 1990 and projected to 2020, vol. 1 . Cambridge: Harvard School of Public Health on behalf of the World Health Organization and the World Bank; 1996.

12. World Bank. World Development Report 1993: investing in health. New York: Oxford University Press; 1993.

13. Ad Hoc Committee on Health Research Relating to Future Intervention Options. Investing in health research and development. Geneva: World Health Organization; 1996.
14. World Health Organization. The World Health Report 1999. World Health Organization 1999;222.

15. Murray CJL, Lopez AD. Alternative projections of mortality and disability by cause 1990-2020: Global Burden of Disease study. Lancet. 1997;349:1498-504.

16. Murray CJL, Lopez AD, Jamison DT. The global burden of disease in 1990: summary results, sensitivity analyses and future directions. Bull World Health Organ. 1994;72:495-508.

17. Murray CJL. Quantifying the burden of disease: the technical basis for disability-adjusted life years. Bull World Health Organ. 1994;72:429-45.

18. Murray CJL, Lopez AD. The incremental effect of age-weighting on YLLs, YLDs, and DALYs: a response. Bull World Health Organ. 1996;74:445-6.

19. Coale A, Guo G. Revised regional model life tables at very low levels of mortality. Popul Index. 1989;55:613-43.

20. WHO. DALY template. 1989. http://www.who.int/healthinfo/ global_burden_disease/tools_national/en/index.html.

21. Development Core Team. A language and environment for statistical computing. Vienna: $\mathrm{R}$ Foundation for Statistical Computing; 2006.

22. Yi Xia, Youlong Gong, Xingyuan Gu. Study on disease burden in Shanghai. Chin J Health Stat. 2008;17:211-4.

23. He J, Gu D, Wu X, et al. Major causes of death among men and women in China. N Engl J Med. 2005;353:1124-34.

24. Goldsmith SK, Pellmar TC, Kleinman AM, Bunney WE, editors. Reducing suicide: a national imperative. Washington, DC: National Academies; 2002.

25. Broome FR, Meixler DB. The TIGER database structure. In: Marx RW (eds) The census bureau's TIGER system, cartography and geographic information systems. American Congress on surveying and mapping: Bethesda, Maryland, vol 17, p. 39-47;1990.

26. Yang G, Hu J, Rao KQ, Ma J, Rao C, Lopez AD. Mortality registration and surveillance in China: history, current situation and challenges. Popul Health Metrics. 2005;3:3. 